\title{
Ocular cysticercosis in a 32-year-old man in Abuja: ultrasonic features as an aid in diagnosis
}

This article was published in the following Dove Press journal:

Clinical Ophthalmology

2 December 2013

Number of times this article has been viewed

\author{
OE Babalola' \\ A Adu ${ }^{2}$ \\ AO Akano ${ }^{3}$ \\ 'Bingham University, New Karu, \\ Nassarawa State, ${ }^{2}$ Department of \\ Ophthalmology, Rachel Eye Center, \\ Garki Phase II, ${ }^{3}$ Department of \\ Radiology, National Hospital, Abuja, \\ Nigeria
}

\begin{abstract}
We report the case of a 32-year-old man suffering from intraocular cysticercosis, with special emphasis on the use of B-scan ultrasound in the diagnosis and management of the condition. An 8000 B-Scan Scanmate was used to obtain the ultrasound images. The patient had worked on a pig farm a few years before presentation. He presented with shadows seen in the right eye. Binocular indirect ophthalmoscopy revealed that he had a choroidal detachment in the right eye inferotemporally. B-scan ultrasound revealed a subretinal subchoroidal cyst with a thick wall containing well defined intracystic echogenic entities representing scolices, and an associated retinal detachment. These findings appear to be pathognomonic. Excision of the cyst through a trans-scleral approach revealed a yellowish serous fluid, with scolices of cysticercus later confirmed histologically. B-scan ultrasound is extremely useful in the diagnosis of ocular cysticercosis and the findings can be pathognomonic.
\end{abstract}

Keywords: ocular cysticercosis, B-scan ultrasound, Nigeria

\section{Introduction}

Cysticercosis is a serious public health problem in a number of developing countries, including Nigeria. ${ }^{1,2}$ About 50 million people worldwide are infected with the taeniasis/cysticercosis complex and 50,000 die from cysticercosis annually. ${ }^{3}$ The typical infected individual is aged 20-50 years. Humans are definitive hosts for Taenia solium ${ }^{4}$ and pigs are the intermediate host. Details of the life cycle of T. solium can be found on the Centers for Disease Control website (http://www. dpd.cdc.gov/dpdx/html/cysticercosis.htm). ${ }^{5}$ In cysticercosis, humans become the intermediate host by ingesting the eggs of $T$. solium from contaminated food and water. As intermediate hosts they cannot excrete the eggs, but remain infected in a dead-end cycle.

The ocular involvement can be destructive because cysticerci may lodge in the subretinal space and sometimes in the intravitreal space. The cysticercus lesion gradually increases in size, and may lead to blindness. Death of the parasite causes release of toxic substances, leading to intense inflammatory reactions and ocular damage. ${ }^{6}$ Initial medical treatment of intraocular cysticercosis with antihelminthic drugs like albendazole or praziquantel has not proven to be particularly useful. Consequently, early surgical removal of the parasite is the treatment of choice..$^{7-9}$

The diagnosis of intraocular cysticercosis is generally only entertained when there is a high index of suspicion or previous experience with it. Typical clinical appearance and supporting investigations such as serology (enzyme-linked immunosorbent assay) 
and B-scan ultrasonography should aid the diagnosis. According to Swastika et al, ${ }^{10}$ it is now possible to carry out mitochondrial DNA analysis on histopathologic sections. Serology by immunoblot is also now possible.

Here we present the case of a 32-year-old man suffering from intraocular cysticercosis, with special emphasis on the use of B-scan ultrasound in the diagnosis and management of the condition. The equipment used to perform ultrasound was a DGH 8000 B-Scan Scanmate (DGH Technology Inc., Exton, PA, USA). The DGH 8000 is a self-contained, portable, multiple mode, ultrasound imaging system. It produces high-resolution video loops and still images with clear, sharp details.

\section{Case report}

A 32-year-old man from Enugu, South Eastern Nigeria, presented to the Rachel Eye Center in Abuja, Federal Capital Territory of Nigeria, with loss of vision affecting the right eye for a period of 2 months. This was described as a "shadow" in that eye, and was associated with a right-sided headache. He had been to a number of eye care facilities where he had been put on prednisolone tablets and cefuroxime capsules with no change in his status. It later transpired that he had once worked on a pig farm soon after his National Youth Service Corps service about 3 years previously.

On examination, his visual acuity was $6 / 4$ and 6/6 R/L, N6 in the right eye and N5 in the left eye. The anterior segment was unremarkable in both eyes. Vitreous reaction in the right eye was $2+$ cells. Fundus examination of the right eye revealed a solid-looking elevation of the retina of 3-4 disc diameters in the inferior temporal quadrant of the retina about 1.5 disc diameters high. There was an associated retinal detachment in the lower half of the retina in the form of "undulating water marks".

\section{Imaging findings}

The B-scan probe was moved from one side of the closed upper eyelid to the other, and some images were acquired by placing the probe on the lower lid as well. B-scan ultrasound in the right eye showed dome-shaped, echogenic, well defined thick membranous structures extending into the vitreous space from the posterior and temporal aspects of the right eye. The membranes were attached in their peripheries to their origins, with their convex sides projecting towards the center of the eye. These membranes were not mobile during the examination. In addition, multiple, small, round echogenic bodies of varying sizes were seen in the submembranous spaces, (later found to be bladder forms of recognizable organisms with suckers). These bodies were also nonmobile.

The overall appearance suggested choroidal detachment with submembranous bodies, later discovered to be from ocular cysticercosis. In addition, some slices (Figure 1E, $\mathrm{G}$, and $\mathrm{H}$ ) showed areas of a rather shallow slightly mobile membranous structure extending into the vitreous space, suggestive of a shallow retinal detachment and consistent with the clinical findings on binocular indirect
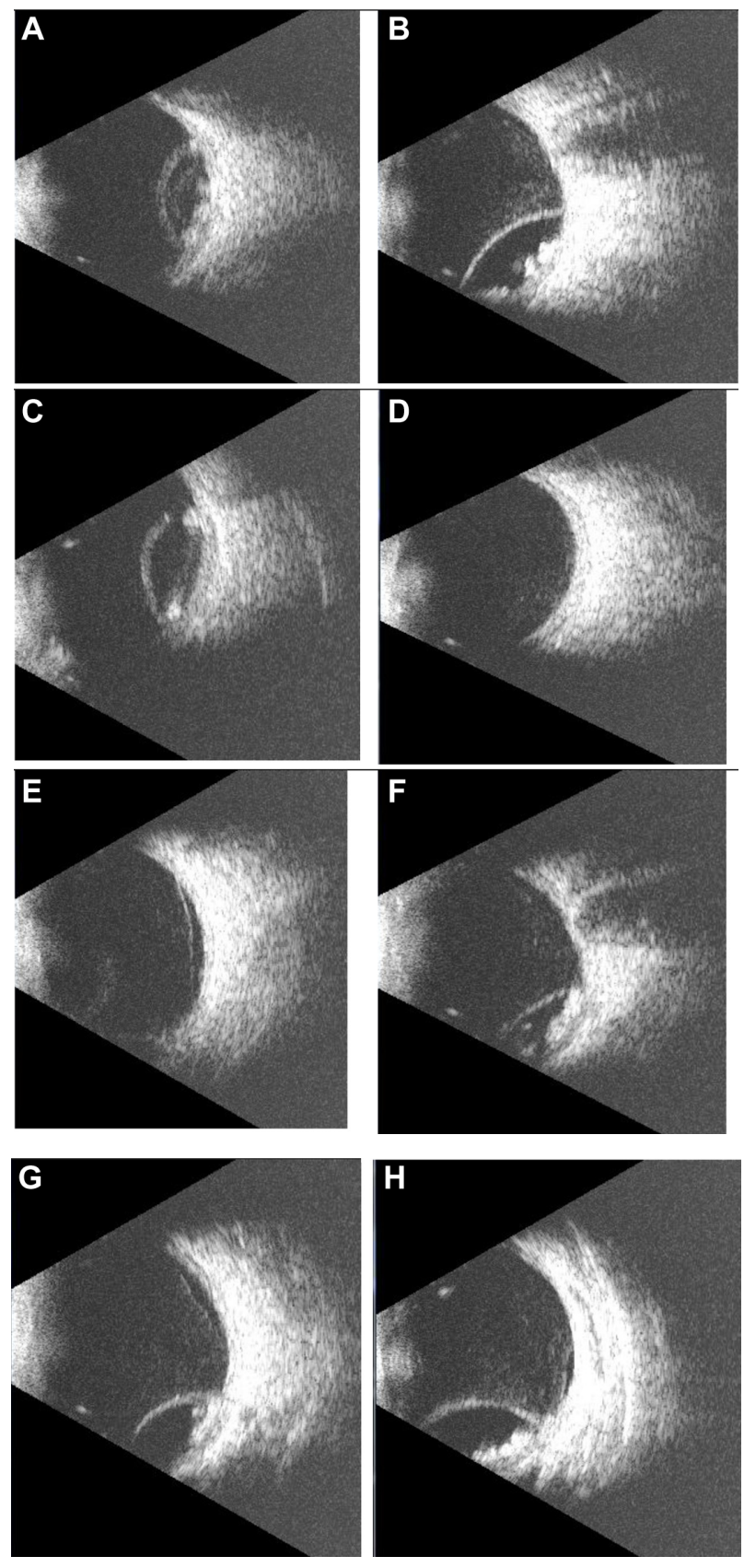

Figure I A series of B-scan slices from the described ocular cysticercosis case. 
ophthalmoscopy. Figure 2 shows the main features of ocular cysticercosis.

\section{Management and follow-up}

Because the main cyst appeared to be a choroidal rather than a retinal detachment, we made the decision to drain the fluid and send it for histopathologic analysis through a radial trans-scleral suprachoroidal incision. Some amber-colored semiviscous liquid was seen. In addition, some cheesy material was also noticed to prolapse from the surgical wound, with subsequent collapse of the choroidal detachment. This was carried out 2 days after presentation. Later on, histologic sections showed a cystic lesion lined by the tegument of an organism. Within the lesions were bladder forms of recognizable organisms with suckers. There was a prominent investing tegument and smooth muscle fibers. The suggested histologic diagnosis was that of ocular cysticercosis.

The patient was subsequently started on albendazole and observed. Over the next few weeks, some retinal hemorrhage was noticed over the surgical site, extending to involve the inferior temporal quarter of the fundus. This was later noticed to resorb slowly, leaving some apparently necrotic areas on the retina inferiorly. At this stage, the patient was lost to follow-up.

\section{Discussion}

\section{Etiology and demographics}

The true distribution of cysticercosis in Nigeria and indeed in other developing tropical countries is broad,

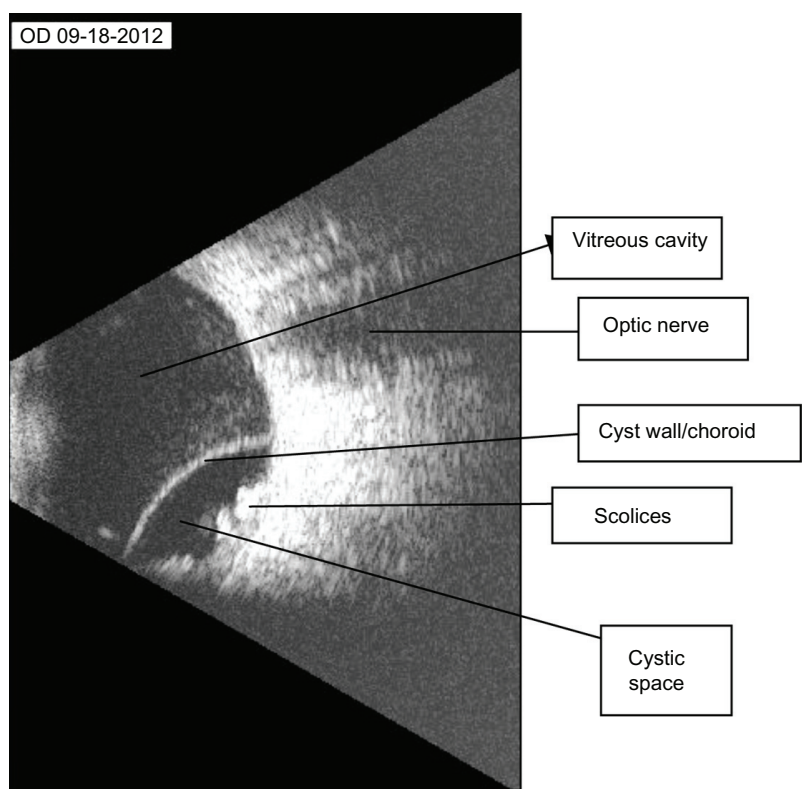

Figure 2 B-scan ultrasound with labels showing the most significant features. with about $40 \%$ of the porcine population in some studies in Nigeria being seropositive for cysticercosis. ${ }^{1}$ One can surmise that seropositivity in the human population will reflect the high rate in pigs. In another region of Nigeria, the prevalence of porcine cysticercosis and human taeniasis was reported to be $20.5 \%$ and $8.6 \%$, respectively. ${ }^{11}$ It is therefore necessary for the ophthalmologist working in an endemic area such as Nigeria to be reasonably familiar with the natural history and possible presentation of this disease.

Ocular cysticercosis can be a serious problem for affected patients because it can easily lead to vision loss, particularly from the toxins released. ${ }^{10}$ Various parts of the eye can be affected, including the periorbital tissue and eyelids, the subconjunctival and sub-Tenon's space, the orbit, the choroid, the suprachoroidal space, the retina, and the vitreous. ${ }^{6}$ In the present case, the choroid and suprachoroidal space were affected, leading to a clinical presentation of choroidal detachment of unknown cause. The true cause of the lesion was not known until the histology report became available. It therefore becomes necessary to have a high index of suspicion.

\section{Clinical and imaging findings}

Karl Dussik, a neurologist and psychiatrist at the University of Vienna, is generally regarded to have been the first physician to use ultrasound for medical diagnosis, particularly of brain tumors, back in 1942. In 1958, Ian Donald pioneered obstetric ultrasound, and Douglas Howry and Joseph Holmes pioneered the development of two-dimensional B-mode ultrasound at the University of Colorado. ${ }^{12}$ There are now applications of B-scan ultrasonography in anesthesia, cardiology (particularly echocardiography and Doppler), pulmonary medicine, general surgery, obstetrics and gynecology and, of course, ophthalmology. ${ }^{13}$

B-scan ultrasound is one means of rapid and reliable diagnosis of this condition, because the properties of choroidal detachment secondary to cysticercosis (and indeed the echo properties of cysticercal cysts in general) are fairly well elucidated and highly suggestive. ${ }^{6,14}$ These include the presence of a highly reflective and smooth-surfaced, domeshaped echo corresponding to the cyst wall, an echoically empty cystic space, and highly reflective intracystic images corresponding to the scolices. ${ }^{11,15}$

Guillory et $\mathrm{al}^{11}$ suggest that where the medium is cloudy, as in some cases of intravitreal disease, B-scan ultrasound could be extremely helpful in establishing the correct diagnosis. 


\section{Treatment and prognosis}

The main remedy for the treatment of retinal and subretinal cysticercosis is surgical excision. ${ }^{10}$ Surgical removal is mandatory in individuals with intraocular cysts. Medical therapy, other than the use of corticosteroids, is not part of treatment. In individuals with uveitis, perioperative corticosteroid administration is recommended. However, for orbital involvement, deep dissection may be problematic.

Extraocular cysts within the orbit, subconjunctival space, and cysts associated with extraocular muscles are a different proposition. Surgical excision of orbital cysts was once considered to be the ideal treatment modality. However, deep orbital dissection and difficulty in completely excising the cyst because of the surrounding inflammatory response increase the chances of postoperative complications (such as decreased vision and diplopia). Treatment of extraocular muscle cysticercosis has undergone a radical change with the successful results of medical alternatives to surgical excision of the cysts. Anthelmintics (eg, albendazole, praziquantel) and oral corticosteroids have been found to be effective. ${ }^{16,17}$

Cysts deep within the orbit are best treated conservatively with a 4-week regimen of oral albendazole $15 \mathrm{mg} / \mathrm{kg} /$ day in conjunction with oral steroids $1.5 \mathrm{mg} / \mathrm{kg} /$ day in a tapering dose over a one-month period. Treatment may increase inflammation as the cyst involutes, leading to worsening clinical states. Thus, concomitant administration of corticosteroids is recommended to avert an inflammatory response. ${ }^{17}$

Resolution of the cyst may take from a few days to months, depending on the density of the surrounding inflammation. Based on the individual's response to medical therapy, another course of medication may be required.

\section{Differential diagnosis}

Other causes of spontaneous choroidal detachment must be considered These will include trauma, central serous choroidopathy, ${ }^{18,19}$ pigment epithelial detachment, posterior scleritis, uveal effusion syndrome, and hypotonic choroidal effusion following glaucoma surgery. However, the ultrasonic signature of these entities is different from that described in this paper. In addition, central serous choroidopathy and pigment epithelial detachment tend to be subfoveal, unlike the off-foveal disposition in most cases of choroidal cysticercosis.

\section{Conclusion}

Human infestation with cysticercosis is common in Nigeria as well as in other tropical countries where porcine hygiene is poor. ${ }^{20,21}$ As a result, ocular involvement is not uncommon. However, this is the first report from Nigeria, of which we are aware. A high index of suspicion is necessary, and hopefully this report should assist in engendering this. Also, the use of B-scan ultrasonography can prove extremely useful in diagnosis because the ultrasonic signature is, to our understanding, virtually pathognomonic. Ocular cysticercosis is not uncommon and it is necessary to have a high index of suspicion. B-scan ultrasound findings can be pathognomonic if the clinician is familiar with the typical presentation.

\section{Acknowledgments}

The authors thank the nursing staff at Rachel Eye Center for their contributions to this research.

\section{Author contributions}

OEB carried out the scans, AA performed the surgery to remove the cyst from the eye, and AOA interpreted the ultrasound findings. All authors contributed to data analysis, drafting and revising the paper.

\section{Disclosure}

The authors have no proprietary interest in any product mentioned in this paper.

\section{References}

1. Weka RP, Ikeh EI. Seroprevalence of cysticercosis and intestinal parasitism in pigs in Jos Metropolis. J Anim Vet Adv. 2009;8:883-887.

2. Garcia HH, Gonzalez AE, Evans CA, Gilman RH. Cysticercosis working group in Peru. Taenia solium cysticercosis. Lancet. 2003;362: 547-556.

3. Centers for Disease Control and Prevention. Recommendations of International Task Force for Disease Eradication (ITFDE). MMWR Recomm Rep. 1993;42:1-38.

4. Hoberg EP. Taenia tapeworms: their biology, evolution and socioeconomic significance. Microbes Infect. 2002;4:859-866.

5. Centers for Disease Control and Prevention. The life cycle of Taenia solium. Available from: http://www.dpd.cdc.gov/dpdx/html/ cysticercosis.htm. Accessed September 22, 2013.

6. Das D, Deka S, Islam S, et al. Neuro and intraocular cysticercosis: a clinicopathological case report. Eye Brain. 2010;2:39-42.

7. Santos R, Chavarria M, Aguirre AE. Failure of medical treatment in two cases of intraocular cysticercosis. Am J Ophthalmol. 1984;97: 249-250.

8. Sharma T, Sinha S, Shah N, et al. Intraocular cysticercosis: clinical characteristic and visual outcome after vitreoretinal surgery. Ophthalmology. 2003;110:996-1004.

9. Gemolotto G. A contribution to surgical treatment of intraocular cysticercosis. Arch Ophthalmol. 1955;59:365-368.

10. Swastika K, Dewiyani CI, Yanagida T, et al. An ocular cysticercosis in Bali, Indonesia caused by Taenia solium Asian genotype. Parasitol Int. 2012;61:378-380.

11. Guillory SL, Zinn KM. Intravitreal cysticercus cellulosae: ultrasonographic and fluorescein angiographic features. Bull NY Acad Med. 1980;56:655-661. 
12. Mt Sinai School of Medicine. The history of ultrasound in medicine. Available from: http:/www.wcume.org/wp-content/uploads/2011/05/ Tsung.pdf. Accessed September 22, 2013.

13. Allen P, Baxter G, Weston M. Clinical Ultrasound. 3rd ed. London, UK: Churchill Livingstone; 2011.

14. Kumar S, Goel R. Diagnostic ultrasonography of the eye. Available from: http://www.aios.org/cme/cmeseries24.pdf. Accessed October 5, 2013.

15. Kaliaperumal S, Rao VA, Parija SC. Cysticercosis of the eye in south India - a case series. Indian J Med Microbiol. 2005;23:227-230.

16. Hashmi MA, Sharma SK, Bera SP, Bibhuti S. Disseminated cysticercus involving the vocal cords. Ann Trop Med Public Health. 2008;1:66-67.

17. Sihota R, Honavar SG. Oral albendazole in the management of extraocular cysticercosis. Br J Ophthalmol. 1994;78:621-623.
18. Gemenetzi M, De Salvo G, Lotery AJ. Central serous chorioretinopathy: an update on pathogenesis and treatment. Eye. 2010;24:1743-1756.

19. Browning DJ. What you should know about choroidal detachments. Available from: http://www.retinareference.com/content/Choroidal $\% 20$ Detachment\%20-\%20djb6-27-2010websiteversion.pdf. Accessed October 5, 2013.

20. Menon-Mehta S, Hampton R. Ocular cysticercosis. Available from: http://emedicine.medscape.com/article/1204683-overview. Accessed October 5, 2013.

21. Onah DN, Chiejina SN. Taenia solium cysticercosis and human taeniasis in the Nsukka area of Enugu State, Nigeria. Ann Trop Med Parasitol. 1995;89:399-407.
Clinical Ophthalmology

\section{Publish your work in this journal}

Clinical Ophthalmology is an international, peer-reviewed journal covering all subspecialties within ophthalmology. Key topics include: Optometry; Visual science; Pharmacology and drug therapy in eye diseases; Basic Sciences; Primary and Secondary eye care; Patien Safety and Quality of Care Improvements. This journal is indexed on

Submit your manuscript here: http://www.dovepress.com/clinical-ophthalmology-journal

\section{Dovepress}

PubMed Central and CAS, and is the official journal of The Society of Clinical Ophthalmology (SCO). The manuscript management system is completely online and includes a very quick and fair peer-review system, which is all easy to use. Visit http://www.dovepress.com/ testimonials.php to read real quotes from published authors. 